\title{
Subcellular Localization of Proteins in the Anaerobic Sulfate Reducer Desulfovibrio vulgaris via SNAP-tag Labeling and Photoconversion.
}

\author{
A. Gorur, ${ }^{*}$ C.M. Leung, ${ }^{*}$ D. Jorgens, ${ }^{*}$ A. Tauscher, ${ }^{*}$ J.P. Remis, ${ }^{*}$ D.A. Ball, ${ }^{*}$ S. Chhabra, ${ }^{*}$ V. \\ Fok, ${ }^{*}$ J.T. Geller, ${ }^{*}$ M.Singer* T.C. Hazen, ${ }^{*}$ T.Juba, ${ }^{* *}$ D. Elias, ${ }^{* *}$ J.Wall, ${ }^{* *}$, M. Biggin, ${ }^{*}$ K.H. \\ Downing, * and M. Auer* \\ * Lawrence Berkeley National Laboratory, Berkeley CA 94720 \\ ** University of Missouri, Columbia MO 65211
}

Systems Biology studies the temporal and spatial 3D distribution of macromolecular complexes with the aim that such knowledge will allow more accurate modeling of biological function and will allow mathematical prediction of cellular behavior. However, in order to accomplish accurate modeling precise knowledge of spatial 3D organization and distribution inside cells is necessary. And while a number of macromolecular complexes may be identified by its 3D structure and molecular characteristics alone, the overwhelming number of proteins will need to be localized using a reporter tag. GFP and its derivatives (XFPs) have been traditionally employed for subcelllar localization using photoconversion approaches, but this approach cannot be taken for obligate anaerobic bacteria, where the intolerance towards oxygen prevents XFP approaches.

As part of the GTL-funded PCAP project (now ENIGMA) genetic tools have been developed for the anaerobe sulfate reducer Desulfovibrio vulgaris that allow the high-throughput generation of taggedprotein mutant strains, with a focus on the commercially available SNAP-tag cell system (New England Biolabs, Ipswich, MA), which is based on a modified O6-alkylguanine-DNA alkyltransferase (AGT) tag, that has a dead-end reaction with a modified O6-benzylguanine (BG) derivative and has been shown to function under anaerobic conditions [1]. After initial challenges with respect to variability, robustness and specificity of the labeling signal we have optimized the labeling.

Over the last year, as a result of the optimized labeling protocol, we now obtain robust labeling of 20 out of 31 SNAP strains. Labeling for 13 strains were confirmed at least five times. We have also successfully performed photoconversion on 5 of these 13 strains, with distinct labeling patterns for different strains. For example, DsrC robustly localizes to the periplasmic portion of the inner membrane, where as a DNA-binding protein localizes to the center of the cell, where the chromosome is located. Two other proteins - Thiosulfate reductase and ATP binding protein were found to be cytoplasmically distributed, whereas a molybdenum transporter was found to locate to the cell periphery.

We judge labeling outcome by 1) SDS gel electrophoresis, followed by direct fluorescence imaging of the gel to address specificity of labeling/confirm expected molecular weight, and subsequent Coomassie analysis to ensure comparable protein levels 2) fluorescence intensity of culture by plate 
reader for statistical sampling (after adjustment for respective cell numbers) and 3) fluorescence microscopy for addressing cell-to-cell signal variation and potential localization patterns. All three assays were usually found to be consistent with one another. While we have been able to improve the efficacy of photoconversion by drastically reducing (eliminating) non-specific binding with our altered labeling protocol, we are currently working on reducing non-specific photoconversion reaction arising occasionally in non-labeled cells.

In addition, we have confirmed the presence of SNAP tagged constructs in three recently cloned E.coli strains under promotor control, and are in the process of utilizing them for evaluating the sensitivity of the photoconversion protocol. Fluorescent Activated Cell Sorting was successfully applied to labeled E.coli cells containing SNAP tagged AtpA protein. Different batches of sorted cells, representing low and high labeling intensity, were re-grown and re-labeled and displayed a labeling efficiency similar to the starter culture, supporting the notion that cell-to-cell differences in labeling reflect difference in protein expression, rather then genetic differences.

References

[1] A.Regoes et al. SNAP-tag mediated live cell labeling as an alternative to GFP in anaerobic organisms. BioTechniques 39, (2005) 809.

[2] This research is supported by the U.S Department of Energy, Office of Science, Office of Biological and Environmental Research, Genomics Program: GTL through contract DE-AC0376SF00098 between Lawrence Berkeley National Laboratory and the U.S. Department of Energy.

FIG.1. Fluorescence labeling of AGT and Tetra-Cys tagged strains in DvH. ReAsH labeling of

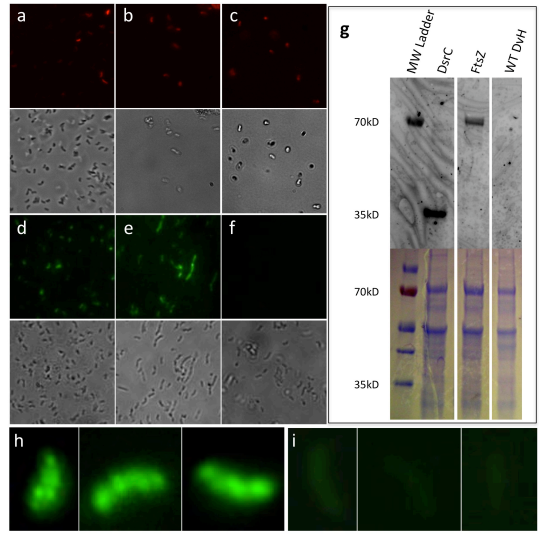

FIG.2. Photoconversion result of 3 different DvH SNAP-tagged strains and wildtype. Note the difference in distribution of photoconversion products. TC-tagged Dsrc (a), WT (b,c) Note that fluorescence signal persists in WT even after extensive washing and addition of excess of EDT (c) suggesting non-specific binding ReAsH fluorophore. SNAP BG-505 labeling of AGT-tagged DsrC (d), FtsZ (e), and WT (f) and corresponding in-gel fluorescence detection and Coomassie-stained SDS PAGE (g). Note the presence of single bands indicating respective appropriate molecular weights, suggesting that SNAP labeling was specific. Close-ups of SNAP-labeled Desulfovibrio strains containing either AGT-tagged ModA (h) or a AGT-STFtagged ModA (i) suggesting that at least for ModA the nature of the tag, and presumably tag-size affects expression labeling pattern.

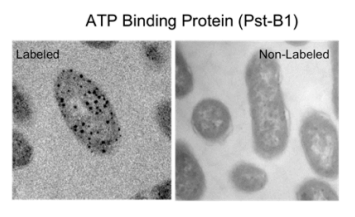

Dissimilatory Sulfite Reductase (Dsrc)

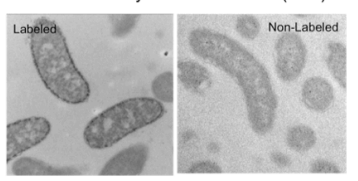

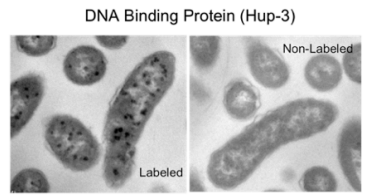

Wild Type DvH

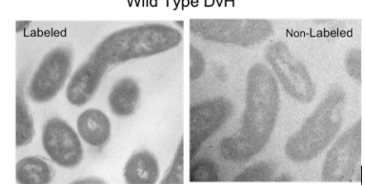

